TTR

Traduction, terminologie, rédaction

\title{
Plaidoyer pour la prise en compte de la spécificité juridique du Québec dans la traduction de contrats
}

\section{Arnaud Tellier-Marcil}

Volume 32, numéro 1, 1er semestre 2019

Traduction et politique(s)

Translation, Politics and Policies

URI : https://id.erudit.org/iderudit/1068018ar

DOI : https://doi.org/10.7202/1068018ar

Aller au sommaire du numéro

\section{Éditeur(s)}

Association canadienne de traductologie

ISSN

0835-8443 (imprimé)

1708-2188 (numérique)

Découvrir la revue

Citer cet article

Tellier-Marcil, A. (2019). Plaidoyer pour la prise en compte de la spécificité juridique du Québec dans la traduction de contrats. TTR, 32(1), 177-204. https://doi.org/10.7202/1068018ar

\section{Résumé de l'article}

Le Québec est une société distincte au sein du Canada en raison de la langue, mais également par sa culture. On y trouve notamment une culture juridique unique. Concrètement, la common law est le régime applicable dans l'ensemble des provinces hormis le Québec, où c'est plutôt le droit civil qui régit les rapports d'ordre privé. La préservation de cette spécificité juridique ne relève pas seulement de la responsabilité des autorités publiques. Les traducteurs de contrats ont aussi un rôle important à jouer. Cependant, nombreux sont ceux qui n'en ont pas conscience et qui se contentent de franciser les termes de common law, une démarche littérale simplifiée par la normalisation de ce vocabulaire, en anglais comme en français. Un tel procédé de nature sourcière est susceptible d'entraîner des conséquences néfastes. D'un point de vue purement matériel, il pourrait s'avérer coûteux pour l'une des parties au contrat en cas de litige, puisqu'il est impossible de savoir comment le tribunal québécois saisi de l'affaire interprétera les termes de common law francisés, qui, dans bien des cas, revêtent un sens distinct ou sont inconnus en droit civil. D’un point de vue politique, les contrats traduits selon une logique sourcière contribuent au phénomène d'acculturation juridique du Québec. En effet, les termes de common law qui sont importés à tort dans les contrats destinés au Québec risquent de s'introduire tôt ou tard dans le langage courant, au détriment du vocabulaire du droit civil. Pour éviter de cautionner ainsi l'anéantissement du système juridique québécois, il suffit d'adopter une méthode cibliste. Plutôt que de se complaire dans la transposition littérale des notions de common law, le traducteur de contrats doit chercher un équivalent fonctionnel dans le lexique du droit civil québécois. La version française ne constituera certes pas un miroir formel de la version anglaise, mais il est normal qu'il en soit ainsi, puisque le système juridique n'est pas le même au Québec que dans les autres provinces canadiennes.
Ce document est protégé par la loi sur le droit d'auteur. L’utilisation des services d'Érudit (y compris la reproduction) est assujettie à sa politique d'utilisation que vous pouvez consulter en ligne.

https://apropos.erudit.org/fr/usagers/politique-dutilisation/ 


\title{
Plaidoyer pour la prise en compte de la spécificité juridique du Québec dans la traduction de contrats
}

\author{
Arnaud Tellier-Marcil \\ Université de Montréal
}

Résumé

Le Québec est une société distincte au sein du Canada en raison de la langue, mais également par sa culture. On y trouve notamment une culture juridique unique. Concrètement, la common law est le régime applicable dans l'ensemble des provinces hormis le Québec, où c'est plutôt le droit civil qui régit les rapports d'ordre privé. La préservation de cette spécificité juridique ne relève pas seulement de la responsabilité des autorités publiques. Les traducteurs de contrats ont aussi un rôle important à jouer. Cependant, nombreux sont ceux qui n'en ont pas conscience et qui se contentent de franciser les termes de common law, une démarche littérale simplifiée par la normalisation de ce vocabulaire, en anglais comme en français. Un tel procédé de nature sourcière est susceptible d'entraîner des conséquences néfastes. D'un point de vue purement matériel, il pourrait s'avérer coûteux pour l'une des parties au contrat en cas de litige, puisqu'il est impossible de savoir comment le tribunal québécois saisi de l'affaire interprétera les termes de common law francisés, qui, dans bien des cas, revêtent un sens distinct ou sont inconnus en droit civil. D'un point de vue politique, les contrats traduits selon une logique sourcière contribuent au phénomène d'acculturation juridique du Québec. En effet, les termes de common law qui sont importés à tort dans les contrats destinés au Québec risquent de s'introduire tôt ou tard dans le langage courant, au détriment du vocabulaire du droit civil. Pour éviter de cautionner ainsi l'anéantissement du système juridique québécois, il suffit d'adopter une méthode cibliste. Plutôt que de se complaire dans la transposition littérale des notions de common law, le traducteur de contrats doit chercher un équivalent fonctionnel dans le lexique du droit civil québécois. La version française ne constituera certes pas un miroir formel de la version anglaise, mais il est normal qu'il en soit ainsi, puisque le système juridique n'est pas le même au Québec que dans les autres provinces canadiennes.

Mots-clés : traduction de contrats, traduction juridique, traduction sourcière, traduction cibliste, équivalent fonctionnel 


\section{Abstract}

Québec is a distinct society within Canada, not only due to its language but also because of its culture. In particular, it has its own legal culture. While the other provinces have a common law system, Québec's private sector is subject to a civil law system. The preservation of this distinct legal system is not the sole responsibility of the government. Contract translators have a role to play as well. However, many of them do not realize it, and restrict themselves to translating literally the common law concepts they encounter, which is particularly straightforward thanks to the normalization of the common law vocabulary, in both English and French. This source-oriented approach may have adverse consequences. From a purely material point of view, it could result in a significant cost for one of the parties in the event of a dispute, since no one knows what meaning the Québec court will give to the common law terms, which in most cases have a different meaning or simply do not exist in the civil law system. From a political point of view, contracts translated with a source-oriented approach contribute to the legal acculturation of Québec. Indeed, if common law terms are used in Québec contracts, they will sooner or later make their way to the everyday language, to the detriment of the civil law vocabulary. To avoid being involved in the destruction of Québec's legal system, one must take a target-oriented approach. Instead of merely translating literally the common law concepts, the contract translator must search for a functional equivalent in the civil law vocabulary. The French version surely will not be a formal mirror of the English version, but it is better that way since the legal system is not the same in Québec as in the other Canadian provinces. Keywords: contract translation, legal translation, source-oriented translation, target-oriented translation, functional equivalent

\section{Introduction}

La traduction juridique revêt une importance vitale en ce qui a trait à la préservation de la spécificité du Québec. Elle joue d'abord un rôle de premier plan dans la valorisation du fait français, le trait distinctif le plus évident de la nation québécoise. Dans un État à prédominance anglophone tel que le Canada, la communauté francophone est condamnée à la marginalité si on ne met pas à sa disposition une version française des textes juridiques qui lui sont applicables.

Le cas de figure le plus flagrant est celui de la non-traduction (Ferreira Duarte, 2000). Le Canada n'est pas étranger à cette pratique, qui a même présidé à la création du pays. De fait, la Loi constitutionnelle de 1867, ciment de la fédération canadienne, a été adoptée exclusivement en anglais. Quelques traductions françaises ont vu le jour, mais elles sont dépourvues de valeur juridique (Tremblay, 2000). En outre, la non-traduction des textes législatifs accable encore aujourd'hui les francophones de certaines provinces canadiennes. L'uni- 
linguisme législatif pratiqué en Alberta s'est d'ailleurs récemment trouvé au cœur de l'actualité, quand la Cour suprême du Canada a rendu un arrêt fatal dans lequel elle niait aux Franco-Albertains le droit à une législation bilingue ${ }^{1}$.

Les politiques de non-traduction ne représentent pas le seul danger d'ordre législatif pesant sur la vitalité de la langue française au Canada. Les traductions de qualité insuffisante ont elles aussi un effet néfaste sur la langue juridique et, par ricochet, sur la langue générale. Jusqu'aux années 1960 environ, la version française des lois fédérales était, de façon générale, d'une médiocrité affligeante, comme l'a souligné maintes fois le jurilinguiste Jean-Claude Gémar (1982, 2005, 2015). Pour s'en convaincre, il suffit de penser au terme "corporation", calqué sur son homographe anglais, qui a longtemps été employé dans les textes de loi dans le sens de "personne morale», un usage totalement incompatible avec la langue française, où il désigne plutôt un corps professionnel (Tellier-Marcil,2016, p. 80-84). Cet anglicisme consacré par la loi a fait de véritables ravages dans le langage juridique et commercial, au point où les juristes l'emploient encore massivement malgré les changements législatifs (Lavoie, 2003).

Le portrait de la traduction juridique au Canada est aujourd'hui bien plus lumineux. La qualité de la version française des lois fédérales s'est améliorée de façon spectaculaire grâce aux progrès remarquables de la jurilinguistique, qui est maintenant digne d'être considérée comme une discipline à part entière (Gémar et Kasirer, 2005). De la même façon, les jugements des tribunaux fédéraux sont désormais traduits en français par des professionnels chevronnés (Gémar, 2015), ce qui se veut une profonde marque de respect pour les citoyens francophones. On peut également se réjouir de la délicatesse dont font preuve les entreprises exerçant des activités au Québec, qui, dans bien des cas, prennent soin de faire traduire leur documentation juridique vers la langue locale, parfois par obligation, en vertu notamment de la Charte de la langue française $e^{2}$, mais souvent aussi de leur plein gré, par égard pour la population francophone.

Ainsi, grâce à des revendications politiques tenaces et aux efforts admirables des traducteurs, une prise de conscience collective s'est progressivement réalisée. L'apport de la traduction juridique dans la sauvegarde de la spécificité linguistique du Québec semble maintenant reconnu. On ne peut toutefois en dire autant de son apport au main-

1. Caron c. Alberta, [2015] 3 RCS 511.

2. Loi québécoise destinée à promouvoir l'usage de la langue française. 
tien de la spécificité juridique de la province. Il s'agit pourtant là aussi d'un héritage d'une valeur inestimable. La survie d'une nation passe bien sûr par la préservation de sa langue, mais également par la valorisation de sa culture. Or, le système juridique d'un peuple fait partie intégrante de sa culture (Popovici, 2009). C'est dans cette optique que s'inscrit le présent article, qui vise à attirer l'attention sur la dimension culturelle de la traduction juridique, un paramètre «trop souvent méconnu» (Sparer, 2002, p. 268).

Avant d'en arriver au plaidoyer annoncé en titre, il est impossible de faire l'économie d'un regard historico-politique sur la situation juridique du Canada (section 1), nécessaire pour comprendre les principaux vecteurs d'acculturation, dont le traducteur de contrats fait partie (section 2). L'analyse se concentre ensuite sur l'enjeu traductologique, pragmatique, culturel et politique que soulève la traduction de contrats, enjeu qui se résume concrètement à un choix entre deux approches théoriques (section 3). Pour compléter le tableau, il est finalement question des techniques que le traducteur de contrats peut adopter en pratique (section 4).

\section{Le bijuridisme bilingue canadien}

À l'époque de la Nouvelle-France, les lois applicables étaient les lois françaises. Les ancêtres des Québécois étaient donc assujettis entièrement à un régime de droit civil. Puis, en 1760, les Britanniques ont conquis le territoire et ils y ont rapidement imposé leur système juridique, un régime de common law. Les francophones se sont opposés vigoureusement à cet anéantissement politique de leur système juridique. Les Britanniques ont finalement cédé en 1774 : en vertu de l'Acte de Québec, le droit français a été rétabli. Il est toutefois important de noter que le retour du droit français, donc du droit civil, nétait que partiel. Il ne concernait que le droit privé. C'est dire que le droit public demeurait sous l'empire du droit anglais, donc de la common law (Arbour, 2012).

C'est sur cette base que s'est construit le Canada actuel, que l'on peut qualifier de bijuridique. Dans toutes les provinces anglophones, le droit applicable, tant dans le domaine public que dans le domaine privé, est la common law d'origine britannique. Dans la seule province francophone, le Québec, c'est également la common law qui chapeaute le droit public, tandis que le droit privé demeure régi par un système d'inspiration française, issu de la tradition romano-civiliste. C'est dire qu'en matière de droit privé, le Québec possède une culture 
juridique unique au Canada, à savoir le droit civil. Ce système juridique est fondamentalement différent de la common law, non seulement par son origine historique, mais également par sa philosophie (Sparer, 1979), par son style (Gémar et Kasirer, 2005), par sa méthode (Touchette, 2002) et par ses concepts (Groffier, 1990). Tout comme la common law est indissociable de la langue anglaise (Baudouin, 1996), l'instrument linguistique avec lequel elle a été façonnée, le droit civil entretient une relation intime avec la langue française, un système linguistique dans lequel il s'exprime naturellement depuis longtemps. Ainsi, le droit civil est une pièce maîtresse de la «langue-culture» (Meschonnic, 1973) du Québec francophone.

Le système politique canadien n'est pas seulement bijuridique, il est également bilingue, une combinaison qui produit des résultats inusités. Le jurilinguiste Gérard Cornu a pu observer quelle "porte au paroxysme la complexité» $(1995$, p. 13). Concrètement, elle divise le Canada en quatre groupes sociolinguistiques : les anglophones soumis à un régime de common law, les francophones assujettis au droit civil, les anglophones soumis au droit civil et les francophones assujettis à la common law (Touchette, 2002, p. 126). Sur le plan terminologique, le langage juridique du Canada se décline donc en quatre vocabulaires distincts, deux en français et deux en anglais (Sparer, 2002, p. 270).

En contexte pancanadien, il est difficile de faire fi du caractère bilingue du pays. Rares sont ceux qui ignorent que les francophones s'attendent à ce que l'on communique avec eux dans leur langue maternelle. Par contre, il arrive fréquemment qu'on oublie ou qu'on feigne d'oublier le caractère civiliste du Québec. C'est le phénomène de l'acculturation juridique, dont les principales sources sont présentées à la prochaine section.

\section{Les sources d'acculturation juridique}

La situation politique particulière du Canada fait en sorte que le droit civil ne constitue qu'un îlot dans un océan de common law. Et s'il n'est pas vigoureusement défendu par les autorités publiques et la société civile, cet îlot pourrait un jour être complètement englouti sous les flots anglo-saxons. En effet, il ne faut pas sous-estimer la pression que la common law fait peser sur le droit civil depuis la conquête britannique. De façon générale, cette pression est exercée, volontairement ou non, par trois grandes catégories d'acteurs: les législateurs (2.1), les tribunaux (2.2) et les professionnels, au premier chef les traducteurs (2.3). 


\subsection{Les législateurs}

La fragilité du droit civil au Canada a pour origine profonde la loi canadienne suprême, à savoir la constitution. Du fait du partage constitutionnel des compétences, le droit civil québécois est relégué à l'arrière-plan au sein de la fédération canadienne. En vertu du paragraphe 92(13) de la constitution de 1867, les provinces se voient certes attribuer le pouvoir de légiférer relativement à la "propriété» et au "droit civil», mais cette habilitation générale est dans les faits fortement entravée par les nombreuses compétences spécifiques réservées au Parlement fédéral, notamment en matière de navigation ${ }^{3}$, d'agriculture ${ }^{4}$, de commerce ${ }^{5}$ et de divorce ${ }^{6}$, et, surtout, par la prérogative fédérale relative à la "paix», à l'« ordre» et au «bon gouvernement » ${ }^{7}$, qui a permis à l'administration centrale d'avoir la mainmise sur l'industrie aéronautique et, dans une large mesure, sur les questions environnementales (Brun, 1993).

Le périmètre réellement occupé par le droit civil s'avère donc plutôt restreint, quoique non négligeable. Dans ce contexte, il est d'autant plus déplorable que, même dans l'enceinte étroite où le droit civil devrait en principe régner, l'influence de la common law se fasse sentir de façon aussi nette. On ne compte plus les concepts de common law qui ont été introduits par le législateur québécois dans des pièces législatives relevant pourtant strictement du droit civil, dont le Code civil lui-même (Normand, 2011). Bien sûr, on ne peut pas condamner tous ces emprunts en bloc. Il est normal que les traditions juridiques s'influencent mutuellement. Ainsi, ce serait donner un coup d'épée dans l'eau que de demander que toute trace du concept de «raisonnabilité» soit effacée du droit civil québécois. Il est vrai que la notion tire son origine de la common law (Gémar et Ho-Thuy, 2016, p. 445), mais son intégration au système civiliste répondait à un besoin réel et a été effectuée dans le respect de l'esprit du droit civil (Tellier-Marcil, 2016, p. 115-118).

\section{Par. 91(10).}

4. L'article 95 prévoit que la compétence est partagée avec les provinces tout en donnant prépondérance aux lois fédérales.

5. Par. 91(2).

6. Par. 91(26). Ainsi, dans le chef d'œuvre d'incohérence que constitue la constitution canadienne, les provinces sont compétentes pour la «célébration» du mariage (par. 92(12)) mais pas pour sa dissolution!

7. Premier alinéa de l'article 91. 
À titre d'exemple d'emprunt condamnable, on peut signaler la Loi sur le transfert de valeurs mobilières et l'obtention de titres intermédiés ${ }^{8}$, adoptée en 2008 dans le but avoué d'harmoniser le droit civil québécois avec la common law canadienne ${ }^{9}$. Concrètement, cette loi injecte dans le système juridique québécois toutes sortes de notions de common law entrant directement en conflit avec le droit civil en vigueur (Cachecho, 2015). Une telle uniformisation des lois applicables dans les différentes provinces canadiennes est perçue comme un signe de progrès par certains groupes de pression, dont la Conférence pour l'harmonisation des lois au Canada, qui ne voient aucun problème à ce que des lois soient "rédigé[es] de façon semblable partout au Canada», leur argument massue, tristement fataliste, étant que le droit civil québécois s'est déjà largement fondu dans la common law (Sabourin, 2013, p. 531-532).

Sur une note plus encourageante, il importe de saluer les efforts déployés par le législateur fédéral afin d'améliorer la compatibilité de la législation canadienne avec le droit civil québécois. L'initiative a pris naissance en 1995, dans le cadre de la Politique sur le bijuridisme législatif, qui pose le principe selon lequel le respect des deux peuples fondateurs du Canada nécessite non seulement une législation bilingue mais également une législation bijuridique. Le législateur fédéral a donc revu sa législation en utilisant la «technique du doublet [, qui] participe en quelque sorte d'une volonté d'assurer l'harmonisation de deux systèmes de droit sans les fusionner en une même législation, mais reflétant la spécificité de chaque système» (LeBel et Le Saunier, 2006, p. 214-215).

Bref, il est capital de garder un œil attentif sur l'activité législative, susceptible d'avoir une incidence majeure - positive ou négative - sur l'intégrité du droit civil et de son langage. Il faut également avoir à l'esprit que la culture juridique distincte du Québec est menacée non seulement par les lois, mais aussi par l'interprétation qu'en font les tribunaux, plus particulièrement la Cour suprême du Canada.

\subsection{La Cour suprême du Canada}

En tant que plus haute instance judiciaire du pays, la Cour suprême du Canada dispose de pouvoirs qui dépassent à bien des égards ceux des législateurs. Il s'agit en principe d'un organe apolitique, mais la

\section{RLRQ, c. T-11.002.}

9. Elle a été rédigée sur le modèle de la Loi uniforme sur le transfert des valeurs mobilières. 
grande portée de ses décisions a qu'on le veuille ou non d'importantes répercussions politiques. Un exemple caractéristique réside dans ses charges contre la Charte de la langue française ${ }^{10}$, l'outil législatif dont le Québec s'est doté pour veiller à la préservation de sa spécificité linguistique. Comme l'a démontré le chercheur Éric Poirier (2014), cette loi québécoise de nature éminemment politique a progressivement été vidée de sa substance au fil des interventions de la Cour suprême.

La spécificité juridique du Québec est elle aussi mise à mal dans certains arrêts rendus par la juridiction canadienne suprême. Durant la période suivant sa constitution en 1875 , la Cour suprême cherchait activement à harmoniser les deux régimes juridiques en vigueur au Canada ou, plus précisément, à imposer la common law à la grandeur du pays (LeBel et Le Saunier, 2006, p. 182-186). Cette période a été qualifiée d'«époque noire de l'uniformisation systématique du droit québécois avec la common law» (Jobin, 2000, p. 27). La situation s'est ensuite améliorée significativement, sous l'impulsion d'une poignée de juges déterminés à veiller à l'intégrité du droit civil, tels que les juges Mignault, Pigeon et Beetz (LeBel et Le Saunier, 2006, p. 186-197). Aujourd'hui, la Cour suprême n'est, en règle générale, plus perçue comme une menace pour le droit civil québécois, mais cette idée reçue ne résiste pas à une analyse de sa jurisprudence (Popovici, 2000).

En effet, la tendance à l'uniformisation de la Cour suprême est loin de s'être volatilisée. À titre illustratif, on peut mentionner l'arrêt Banque nationale de Grèce c. Katsikonouris ${ }^{11}$, que le professeur Adrian Popovici a critiqué avec raison :

le banc [sic] était composé de cinq juges, trois de common law et les juges L'Heureux-Dubé et Gonthier. C'était une question d'assurance et de mandat. Les deux juges québécois se sont retrouvés dissidents, à juste titre : est-ce que les fausses représentations $[$ sic $]$ d'un mandataire lient le mandant? En droit civil, oui. C'est peut-être différent ailleurs... mais... (Popovici, 2000, p. 613)

Il arrive même aux magistrats de la Cour suprême d'admettre à mots à peine couverts leur penchant pour le rapprochement du droit civil et de la common law. Pas plus tard qu'en décembre 2016, le juge Wagner, qui a depuis été nommé juge en chef, écrivait ce qui suit dans l'arrêt Groupe Jean Coutu inc. c. Canada (Procureur général) ${ }^{12}$ : «la convergence

10. RLRQc. C-11.

11. [1990] 2 R.C.S. 1029.

12. [2016] 2 RCS 670. 
naturelle des principes et des résultats [...] est généralement souhaitable ${ }^{13}$. L'époque où les différences culturelles opposant les deux régimes étaient vues, parmi les plus hauts magistrats du pays, comme des anomalies plutôt que comme des richesses n'est donc pas tout à fait révolue...

On peut constater que l'État contribue à l'acculturation juridique du Québec tant par son appareil législatif que par son bras judiciaire. Ce phénomène est régulièrement mis en lumière. On ne peut toutefois pas en dire autant du rôle des professionnels du secteur privé, dont celui des traducteurs.

\subsection{Les traducteurs}

Le droit, «science subordonnée à l'écrit» (Debruche, 2015, p. 30), est indissociable de la langue. Un système juridique, c'est avant tout un langage. Les notions caractéristiques d'une tradition juridique font corps avec les mots utilisés pour les exprimer. Pour que les différents intervenants de la communauté juridique puissent forger une science, ou du moins une branche du savoir à proprement parler, il a fallu élaborer un ensemble de "termes techniques de précision" (Cornu, 2005). La riche pensée civiliste que l'on connaît serait inconcevable sans le lexique unique qui s'est construit ainsi au fil du temps. Bref, ce qui distingue le droit civil des autres régimes juridiques, c'est en grande partie son vocabulaire.

Le rayonnement du droit civil repose donc dans une large mesure sur la diffusion du vocabulaire qui lui est propre. Lorsque les termes français du droit civil sont délaissés au profit de termes issus d'un autre système linguistico-juridique - la common law anglaise en l'occurrence -, la culture civiliste en souffre inévitablement. C'est ainsi que «les mots peuvent contribuer au phénomène d'acculturation» (Moore, 2007, p. 314). Le rôle crucial des gardiens des mots que sont les traducteurs paraît dès lors aller de soi.

La part de responsabilité des traducteurs juridiques dans la mise en valeur du droit civil québécois ne doit pas être sous-estimée. Qu’ils le veuillent ou non, ils sont au cœur de l'action. On pense spontanément aux traducteurs de lois, mais les traducteurs de contrats ne sont pas en reste. De fait, dans le cours de leurs affaires, les grandes entreprises canadiennes ou internationales doivent constamment faire traduire leurs contrats vers le français pour les besoins du marché québécois.

13. Par. 52. 
Or, le contrat constitue l'un des principaux véhicules d'expression du droit privé. C'est donc un domaine où, au Québec, la terminologie du droit civil devrait régner en maître. Ce n'est toutefois malheureusement pas le cas en pratique. Dans une quantité astronomique de contrats traduits, les termes de common law sont rendus non pas par des équivalents conformes au droit civil, comme il se devrait, mais plutôt par des termes de common law francisés. À titre illustratif, les termes de common law «gross negligence» et «wilful misconduct» sont bien souvent rendus respectivement par les calques «négligence grossière» et «inconduite délibérée » plutôt que par les équivalents naturels «faute lourde» (Reid, 2016) et "faute intentionnelle» (Tellier-Marcil, 2016, p. 106-108). Le traducteur qui procède de cette façon ne se rend sans doute pas compte des conséquences sociopolitiques qui en découlent. Il se fait néanmoins malgré lui complice de l'engloutissement du droit civil par la common law. D'où la nécessité d'examiner la traduction de contrats dans une perspective sociale mais pragmatique, qui tient compte de la réalité professionnelle du traducteur.

\section{L'alternative du traducteur de contrats}

Pour comprendre adéquatement l'enjeu terminologique que pose la traduction de contrats au Canada, il importe d'avoir le contexte global à l'esprit. En pratique, la version anglaise de départ n'est habituellement qu'un contrat type, soit un modèle standard qu'une entreprise utilise dans ses relations commerciales en l'adaptant au besoin en fonction de la situation en cause ou des demandes de son cocontractant.

En lui-même, ce contrat type ne reflète l'intention d'aucune partie en particulier; il est seulement le fruit de la volonté générale de l'entreprise de protéger ses intérêts, qui demeurent hypothétiques à ce stade. Lorsqu'il entre en jeu, le traducteur n'est donc pas appelé à relater une relation contractuelle passée, c'est-à-dire à reproduire l'«intention des parties », mais au contraire à créer un nouveau modèle qui servira de base à des relations contractuelles futures.

En termes traductologiques, la version française - habituellement destinée au Québec, il va sans dire - ne sera que rarement une simple «traduction-document», c'est-à-dire une traduction dont «l'objectif est de renseigner le destinataire sur le texte de départ» (Salinas, 2010, p. 288, paraphrasant Nord, 1997). En raison de la nature normative du contrat, il s'agira plutôt en règle générale d'une véritable «traductioninstrument», c'est-à-dire d'une traduction "qui a pour but de fournir le support d'un nouvel acte de communication»(ibid.). Une fois le 
processus de traduction terminé, la version anglaise ne présentera plus guère d'intérêt, car c'est sur la version française que les parties apposeront leur signature. Celles-ci ignoreront d'ailleurs bien souvent que le contrat constitue une traduction.

Cette traduction-instrument, devenue instrument tout court, sera dans la majorité des cas soumise au système juridique local, à savoir le droit civil québécois. Dans des circonstances particulières, il arrive que le contrat exclue expressément le droit québécois en lui substituant un droit étranger. La validité d'une telle stipulation est reconnue de façon générale par l'article 3111 du Code civil du Québec, dont la portée est toutefois tempérée par de nombreuses exceptions visant à protéger les parties vulnérables, par exemple les consommateurs (art. 3117) et les salariés (art. 3118).

En somme, le contexte de traduction peut certes différer d'un contrat à l'autre, mais il demeure possible d'affirmer sans tomber dans la généralisation abusive que, d'ordinaire, les contrats traduits constitueront des traductions-instruments régis par le droit civil québécois. En tout état de cause, l'analyse qui suit s'applique spécialement à ce cas de figure.

\subsection{La dichotomie sourcier/cibliste}

D'un point de vue pratique, le traducteur de contrats confronté à une notion de common law a deux options : soit il importe cette notion de common law dans la version française, soit il lui substitue une notion de droit civil. Sur le plan théorique, ces deux approches antagonistes peuvent être qualifiées respectivement de «sourcière» et de «cibliste». Jean Delisle et Marco A. Fiola définissent la première comme

la manière de rendre le texte de départ dans une forme qui en reproduit le plus possible la lettre et qui importe dans le texte traduit un nombre variable d'éléments linguistiques, culturels et civilisationnels propres au texte de départ. (2013, p. 682)

Ils définissent par ailleurs la seconde comme

la manière de rendre le texte de départ dans une forme qui est la plus naturelle possible pour le lecteur du texte d'arrivée en fonction des usages et conventions de la langue et de la culture d'arrivée. (ibid., p. 646)

L'idée d'opposer les deux approches, «aussi ancienne qu'évidente»(Gémar, 2016, p. 534), aurait été introduite par le philosophe allemand Friedrich Schleiermacher, décédé en 1834 (ibid.), mais les termes «sourcier» et «cibliste» sont une création de Jean-René 
Ladmiral (2014 [1986]). La dichotomie conceptuelle proposée par cet auteur n'a pas manqué de nourrir les réflexions traductologiques. Elle a donné lieu à de jolies images, comme celles de Riccardo Raimondo (2016), qui a poétiquement associé les approches sourcière et cibliste à des personnages issus de la mythologie grecque, respectivement Hermès et Orphée. Elle a également, et surtout, fait l'objet de débats animés. Ladmiral lui-même a publié plusieurs textes pour exprimer sa position, résolument cibliste. S'opposant à la «sacralisation de la langue-source» (2014 [1990], p. 269), il tire à boulets rouges sur les théoriciens à tendance sourcière. Ce faisant, il ne cesse d'utiliser la même métaphore filée, dont le mauvais goût a été dénoncé avec raison par Sherry Simon (1989), mais dont le propos sous-jacent ne manque toutefois pas de pertinence. En effet, Ladmiral n'a pas tort d'inviter les traducteurs à se méfier de l'obsession de la fidélité au texte de départ, qui conduit souvent à négliger les impératifs et les richesses de la langue cible.

Cette opposition entre sourcier et cibliste, aussi commode soitelle, n'est pas à l'abri des critiques. Il est vrai que cette «dichotomie simpliste» (Cordonnier, 2002, p. 49) échoue à rendre compte de toutes les nuances inhérentes à l'activité traduisante (Meschonnic, 2004). En effet, nombreuses sont les traductions de qualité qui ne sont ni sourcières ni ciblistes, ou qui présentent un caractère mixte. Or, Ladmiral n'admet pas cette possibilité. Pour lui, «il faut nécessairement faire un choix : on sera sourcier ou cibliste, mais pas les deux à la fois» (2014, p. XI). L'affirmation paraît nettement trop catégorique dans une perspective traductologique générale, mais force est d'admettre qu'elle s'établit comme une vérité absolue en ce qui concerne l'enjeu spécifique de la traduction en français des termes de common law dans un contrat destiné au Québec. Car cet enjeu, aussi complexe soit-il, se décline forcément en une opposition binaire entre deux solutions terminologiques : soit on utilise une version francisée du vocabulaire de la langue source (common law), soit on puise dans le lexique de la langue cible (droit civil). Il n'y a pas de troisième voie.

Jean Delisle établit une distinction intéressante entre les textes littéraires et les textes pragmatiques. Il observe que cette deuxième "grande catégorie de textes donne lieu généralement aujourd'hui à des traductions ciblistes» (2001, p. 221) et ajoute que "[1]es lois de la communication et le bon sens l'exigent» (ibid.). Ladmiral abonde dans le même sens, allant jusqu'à affirmer que tous les traducteurs techniques sont ciblistes (2014 [1991], p. 233). Cette assertion ne 
correspond toutefois pas à la pratique en matière de traduction de contrats, où la tendance sourcière est très forte.

\subsection{La tendance sourcière}

Les contrats qui circulent dans le monde des affaires et de la consommation se ressemblent de plus en plus, car ils s'inspirent des mêmes modèles, «rédigés par les grands cabinets américains et britanniques dans l'une ou l'autre de leurs succursales [et] utilisés sur tous les continents» (Dalphond, 2003, p. 97). Évidemment, ces modèles s'inscrivent dans la logique de la common law, de sorte que «la mondialisation du commerce entraîne actuellement l'utilisation à travers le monde de concepts développés par la common law» (ibid.). Les versions françaises des contrats applicables au Québec n'échappent pas à cette uniformisation massive. Il serait bien sûr souhaitable qu'une étude empirique soit réalisée pour mesurer l'ampleur exacte du phénomène, mais celui-ci est suffisamment sérieux (ibid.) pour qu'on se préoccupe sans attendre des problèmes de fond qu'il soulève. Pour s'en convaincre, il suffit de jeter un coup d'œil aux contrats d'adhésion qui pullulent sur Internet. Ceux-ci sont d'ailleurs fréquemment intitulés "Termes et conditions", expression calquée sur la formulation utilisée en common law (Gémar et Ho-Thuy, 2016, p. 615). Inutile de préciser que le contenu d'un tel contrat est à l'image de son titre...

La nature sourcière de ces contrats saute donc aux yeux. Le traducteur transpose, consciemment ou inconsciemment, la culture de la common law dans la version française, pourtant régie en principe par le droit civil. Ce penchant pour la traduction sourcière s'explique principalement par la pression qu'exercent le littéralisme et la normalisation sur le traducteur.

\subsubsection{Littéralisme}

Dans quelle mesure le texte cible doit-il être fidèle à la forme littérale du texte source? C'est là «la question traductologique par excellence» (Ladmiral, 2014 [1998], p. 175). D’un point de vue sourcier, le texte traduit doit reproduire autant que possible les caractéristiques linguistiques du texte original (Delisle et Fiola, 2013). Cette approche littérale présente manifestement un attrait puissant pour les traducteurs juridiques. En effet, comme l'a noté Vassilis Koutsivitis, ceux-ci ont une forte propension à utiliser le procédé dit de la «transposition littérale» (1990, p. 226). Différentes raisons peuvent être avancées pour expliquer cet état de fait. 
Tout d'abord, l'apparente complexité du droit, une science non exacte dont la traduction est considérée comme une "tâche ardue» (Monjean-Decaudin, 2010, p. 706), incite de nombreux traducteurs à se réfugier dans le confort de la littéralité. Gémar en est venu à une conclusion similaire :

Le langage du droit occupe une place particulière dans l'imaginaire du traducteur. Pour le commun des mortels, qu'il intimide, le droit est un domaine tellement vaste et complexe que seuls des initiés peuvent s'y risquer. (2002, p. 163)

Ainsi, les traducteurs de contrats qui ne s'estiment pas suffisamment ferrés en droit font preuve d'une extrême prudence et pensent réduire les risques d'infidélité en traduisant mot à mot. Leur souci de fidélité les pousse finalement à verser dans la "traduction automatique» (Reed, 1979, p. 95).

La pression littéraliste vient également des directives reçues par les traducteurs juridiques. D'une part, les juristes avec qui ils travaillent "ont pour habitude de [leur] dire "traduisez mot à mot et laissez le travail d'interprétation aux juristes"»(Salinas, 2010, p. 299). D'autre part, une vision sourcière leur est inculquée dans les cours de traduction juridique. On les encourage parfois directement à adopter une approche littérale, à "traduire non pas l'idée mais les mots" (Després, 2007, p. 6). De même, on leur demande de demeurer dans le système juridique de la langue source et, par voie de conséquence, de calquer les notions juridiques au lieu de les adapter à la culture cible. C'est notamment ce qu'enseigne Michel Sparer :

Ainsi quand on traduit de l'anglais au français, on change de langue mais on ne change pas de système juridique. On n'aboutira pas à faire d'un texte de common law rédigé en anglais un texte de droit civil rédigé en français. (2002, p. 267)

Ce précepte général, enseigné par Sparer et sans doute par bon nombre de ses homologues, est hautement problématique s'il est assimilé sans nuance par les étudiants. S'il peut être appliqué sans réserve à la traduction législative, il s'avère trompeur pour les nombreux traducteurs évoluant dans le secteur privé. De fait, contrairement à ce que Sparer soutient dans son article (p. 271), son raisonnement ne peut que rarement être transposé à la traduction d'actes privés ${ }^{14}$.

En somme, la tendance littéraliste des traducteurs de contrats est attribuable tant à leur crainte de déformer le texte source qu'aux

14. Voir les propos introduisant la section 3. 
directives qu'ils reçoivent. En pratique, il leur serait toutefois plus difficile de verser dans le littéralisme si le vocabulaire de la common law n'avait pas été normalisé.

\subsubsection{Normalisation}

À l'heure de la mondialisation, la normalisation prend de l'ampleur. Elle touche notamment la terminologie, «devenue une priorité de prime importance dans les efforts de normalisation des organisations nationales et internationales»(Gomez et Pinto, 2001, p. 569). Le Canada est particulièrement actif dans le domaine de la normalisation terminologique. Le produit phare de l'administration fédérale en la matière, la banque de données terminologiques TERMIUM Plus (Gouvernerment du Canada, n. d.), représente une véritable mine d'information pour les traducteurs en quête du mot juste.

La recherche d'un vocabulaire normalisé vient à faire partie intégrante du processus de traduction, puisque le traducteur sait qu'«il se trouve le plus souvent face à des notions qui ont déjà été uniformisées dans une perspective translinguistique» (Dullion, 2014, p. 638). Dans le monde du droit, où la norme règne en maittre, la recherche d'une solution normalisée a des allures de dogme. En témoigne cet enseignement de Gémar: «le traducteur et le terminologue ne devraient fonder leur recherche de l'information juridique que sur des éléments à valeur normative» (1980, p. 136).

Jusqu'à récemment, le vocabulaire de la common law, anglophone par essence, n'avait pas de pendant francophone normalisé. Puis, les chercheurs ont été invités à constituer un lexique de common law bilingue (Reed, 1979, p. 97). L'appel à l'uniformisation terminologique a été entendu. Compte tenu de l'importance accordée à la traduction juridique, qui est «un enjeu au plan de la symbolique, mais aussi au plan des batailles et des revendications politiques" (Sparer, 2002, p.271), l'administration fédérale a accepté d'investir dans la normalisation du vocabulaire de la common law, au bénéfice des francophones établis hors Québec. Le Programme national de l'administration de la justice dans les deux langues officielles (PAJLO) a alors vu le jour, donnant ultimement naissance à un vocabulaire de common law bilingue normalisé.

Du fait de son intégration à la banque de données TERMIUM Plus, si aisément accessible, ce vocabulaire normalisé semble abondamment utilisé par les traducteurs de contrats. Il leur offre une solution facile et rapide, tout en les réconfortant par son caractère 
officiel. C'est ainsi que la normalisation a pour effet de marquer de nombreux contrats traduits d'une empreinte sourcière. Le traducteur se croit obligé d'employer la version française normalisée des termes de common law qu'il rencontre, et il ne songe pas à puiser dans la terminologie de la culture juridique cible, à savoir le droit civil québécois.

Autrement dit, la normalisation terminologique effectuée dans le cadre du PAJLO fait «apparaître les contextes de plurilinguisme officiel comme une situation simplifiée, demandant principalement aux traducteurs la maîtrise d'outils spécifiques» (Dullion, 2014, p.650). Elle donne aux traducteurs juridiques l'illusion qu'il leur suffit de maîtriser la banque de données du PAJLO pour résoudre les difficultés posées par les termes de common law. Or, la solution se trouve bien souvent ailleurs.

\subsection{La solution cibliste}

En matière de traduction de contrats, l'approche sourcière a des répercussions néfastes, tant d'un point de vue pragmatique que d'un point de vue politique. Par contraste, la méthode cibliste s'impose comme la voie à suivre. À l'échelle individuelle, elle sert les intérêts économiques du client et, à l'échelle sociale, elle profite ultimement à la collectivité.

\subsubsection{Justifications pragmatiques}

Le contrat n'a rien d'un texte ordinaire. À l'image de la loi (Sparer, 2002, p. 267), il crée des droits et des obligations pour les parties (Gémar, 1988, p. 305), ce qui n'est pas banal. Le traducteur ne peut faire abstraction de cette particularité. Il doit garder à l'esprit que le texte qu'il produit est susceptible, advenant un différend relatif au sens à donner aux clauses du contrat, d'être décortiqué dans le cadre d'un processus judiciaire (Harvey, 2002, p. 178). L'issue de ce processus pourrait fort bien être déterminée par les choix terminologiques effectués par le traducteur. Si celui-ci s'est laissé guider par une logique sourcière, l'interprétation du contrat risque de poser problème. En effet, le tribunal québécois saisi de l'affaire aura de la difficulté à interpréter les termes relevant de la common law francisée, qui, dans bien des cas, revêtent un sens distinct ou sont tout simplement inconnus en droit civil. Et l'interprétation retenue pourrait s'avérer coûteuse pour l'une des parties. 
Prenons comme premier exemple "real property", terme de common law qui désigne les terrains ainsi que les immeubles qui y sont érigés (Garner et Black, 2014). Dans le cadre de la francisation de la terminologie de la common law, l'équivalent qui a été retenu est le calque «bien réel» (Vanderlinden, Snow et Poirier, 2017). Or, il se trouve que, dans le langage du droit civil, l'adjectif «réel» englobe non seulement les terrains et les immeubles, mais également tous les meubles de quelque nature que ce soit (Reid, 2016, «droit réel»)... Celui qui s'engage à vendre «ses biens réels» contracte donc une obligation beaucoup plus large que celui qui s'engage à vendre «his real property».

Le cas de «joint and several», un concept de common law utilisé afin de permettre au créancier d'exiger d'un seul des débiteurs le paiement de l'ensemble de la dette commune, illustre lui aussi l'importance de tenir compte de la culture juridique cible. Plusieurs sources terminologiques bilingues proposent l'équivalent «conjoint et solidaire", que les traducteurs de contrats sont naturellement nombreux à emprunter. Cette solution est toutefois incompatible avec le droit civil. Elle constitue même un non-sens dans ce régime juridique, où une obligation peut être soit conjointe, soit solidaire, mais jamais les deux à la fois. En droit civil, l'obligation conjointe signifie que chaque débiteur n'est tenu qu'au paiement de sa part dans la dette commune (article 1518 du Code civil du Québec), ce qui est précisément le contraire de l'obligation «joint and several», correspondant plutôt à l'obligation «solidaire» du droit civil (art. 1523) ${ }^{15}$.

$\mathrm{Nul}$ besoin de préciser que les conséquences pécuniaires d'une traduction sourcière de ce type peuvent se révéler catastrophiques pour l'une des parties. La réputation professionnelle du traducteur pourrait également s'en trouver entachée. Ces considérations matérielles ne sont pas négligeables, mais au-delà de celles-ci, ce sont des motifs éminemment politiques qui militent en faveur d'une traduction respectueuse de la tradition juridique québécoise.

\subsubsection{Justifications sociopolitiques}

C'est sans contredit dans l'arène politique que se déroule l'essentiel du combat entre la méthode sourcière et la méthode cibliste. De part et d'autre, les attaques sont virulentes. D'un côté, Henri Meschonnic et Antoine Berman, deux auteurs pouvant être rangés dans le camp

15. Sur cette question, voir Tellier-Marcil (2016, p. 111-114). 
des sourciers, accusent les ciblistes de colonialisme linguistique (Meschonnic, selon Ladmiral, 2014 [1990], p. 263) ou d'ethnocentrisme (Berman, 1984). Ils dénoncent la «négation systématique de l'étrangeté de l'œuvre étrangère» (ibid., p. 17). À titre illustratif, dans les traductions bibliques de Meschonnic, le nom de Dieu n'est pas traduit en français. Adonaï reste Adonaï, ce qui favorise l'immersion du lecteur dans la culture hébraïque. D’un point de vue sourcier, Meschonnic évite ainsi l'engloutissement de la culture source par la culture cible. La critique est valable, mais le raisonnement inverse l'est tout autant. Il peut certes paraître ethnocentrique de raconter les récits bibliques dans une perspective française (culture cible), mais il en va de même d'une narration centrée sur la perspective des Hébreux (culture source). Il s'agit alors d'une «hébraïsation du français» (Ladmiral, 2014 [1998], p. 193), ce qui est aussi une forme de colonialisme linguistique. Pour sortir de l'impasse, il faut d'abord reconnaitre que l'ethnocentrisme est une fatalité, puis élargir quelque peu la réflexion à la manière de Jean-Louis Cordonnier :

Il est vrai que toutes les cultures sont plus ou moins ethnocentriques, à la différence près, et qui est quand même de taille, que certaines d'entre elles dominent alors que d'autres non. (2002, p. 47)

Le constat frappe par sa lucidité. Il met en évidence l'essentiel, qui est de protéger les cultures vulnérables contre le colonialisme rampant des cultures dominantes.

Lorsque le débat se transporte dans le monde juridique, le spectre de l'ethnocentrisme prend la forme plus précise de «juricentrisme». Sylvie Monjean-Decodin définit l'approche juricentriste en ces termes défavorables :

Pour un traducteur, le «juricentrisme» consiste à traduire coûte que coûte, au détriment de la culture juridique source, un terme ou un concept sans équivalence par un terme ou concept propre à son droit et à sa langue. Il s'agit de tirer à soi le droit étranger pour l'amener vers la terminologie ou le concept de son propre système de pensée juridique, dénaturant à la fois le droit source et la traduction. (2010, p. 704)

L'auteure se présente ici comme une sourcière dénonçant les traductions pensées en fonction de la culture juridique cible. Il ne s'agit que d'une critique générale, mais si elle était formulée dans le contexte de la traduction vers le français de contrats anglo-canadiens, elle serait nettement abusive, car il est injuste de condamner celui qui veut protéger un droit civil se noyant de plus en plus dans la common law. 
Monjean-Decodin est d'ailleurs elle-même consciente du danger : "L'indiscutable suprématie de l'anglais risque d'entraîner une uniformisation culturelle, donc un appauvrissement du patrimoine culturel mondial»(ibid., p. 706). Or, dans le contexte canadien, il est impossible de veiller à la préservation de la diversité juridicoculturelle sans faire preuve de juricentrisme. Le traducteur de contrats qui évacue les concepts de common law au profit de notions appartenant à son propre régime juridique, le droit civil, pourra certes être accusé de juricentrisme, mais il aura choisi le moindre mal, car il aura dit non à la colonisation du droit civil québécois par la pensée juridique anglo-saxonne.

C'est donc d'abord pour un motif politique que l'approche cibliste devrait être privilégiée par le traducteur de contrats. En reproduisant les concepts canado-britanniques dans les contrats utilisés au Québec, le traducteur sourcier se trouve à cautionner la domination de l'empire de "Sa Majesté du chef du Canada», pour reprendre ironiquement cette expression singulière qui pullule tant dans les lois fédérales que dans les lois du Québec ${ }^{16}$. Quant à lui, le traducteur cibliste, adaptant son texte à la langue-culture cible, unique en Amérique du Nord et de ce fait spécialement vulnérable, se positionne d'une certaine façon contre l'uniformisation juridicopolitique du Canada, et pour le respect de la société distincte que constitue le Québec. Cette prise de position implicite est facile à assumer puisqu'elle ne prête guère à controverse. Comme le contrat original était adapté à la culture juridique de ses propres lecteurs, la common law, il est légitime que la traduction soit elle aussi adaptée à ses lecteurs et à leur culture, en l'occurrence la culture civiliste.

En faisant l'effort de réexprimer les concepts de common law dans un langage compatible avec la langue-culture cible, les traducteurs protègent la société tout entière contre une acculturation juridicolinguistique. En effet, les termes de common law qui sont importés à tort dans les contrats québécois finissent tôt ou tard par s'introduire également dans le langage courant. À terme, la population québécoise aura oublié les concepts de droit civil, qui seront remplacés dans leur esprit par des notions de common law.

Malheureusement, ce processus d'acculturation par la traduction de contrats est déjà enclenché. Pour en témoigner, on peut citer l'exemple de l'introduction rampante dans l'usage d'une acception impropre du terme "intérêt», calquée sur le sens attribué à son quasi-

16. Voir par exemple la Loi sur la taxe de vente du Québec, RLRQ c T-0.1. 
homographe «interest» en common law. Dans ce régime juridique, un interest est "a comprehensive term to describe any right, claim, or privilege that an individual has toward real or personal property" (Lehman et Phelps, 2005). La plupart des ressources terminologiques, TERMIUM Plus en tête, pointent vers le calque "intérêt» pour rendre ce concept anglo-saxon. Dans ce contexte, il n'est pas étonnant que les traducteurs de contrats aient massivement recours à cette solution facile. Celle-ci ne respecte pourtant aucunement la logique du droit civil. En effet, comme l'ont noté avec justesse Gémar et HoThuy, «en droit civil, on ne connaît pas de catégorie de droits appelée "intérêt"»(2016, p. 298). Pour désigner ce que les anglophones appellent «interest», on utilise plutôt l'expression «droit réel», ou tout simplement le mot «droit», qui convient parfaitement dans la plupart des cas (Tellier-Marcil, 2016, p.108-111). Du fait notamment de la répétition continuelle du terme fautif «intérêt» dans les contrats traduits, les francophones sont toutefois aujourd'hui nombreux à parler spontanément d'un intérêt dans un bien plutôt que d'un droit dans un bien.

Ces erreurs terminologiques commises sous l'influence de la common law sont bien plus pernicieuses que de simples anglicismes. Il s'agit non seulement d'emprunts inutiles à une autre langue, mais également d'emprunts inutiles à une autre culture juridique. Au risque d'anglicisation, bien connu, s'ajoute donc le risque d'acculturation juridique, moins connu mais tout aussi préoccupant. À cet égard, il faut saluer les nombreux efforts déployés, à juste titre, pour préserver la langue française, mais on ne peut que déplorer le peu d'intérêt manifesté pour la préservation du régime juridique qui y est associé.

La survie du droit civil au Canada dépend de nombreux facteurs, dont plusieurs relèvent certes des plus hautes instances décisionnelles, mais au nombre desquels figure également le langage des contrats. Comme le nombre de contrats d'abord rédigés en anglais est incommensurable, leur traduction systématique vers une common law francisée (traduction sourcière) contribuerait grandement à l'assimilation juridique des Québécois. La common law francisée peut faire des ravages si elle se fraie un chemin dans l'usage général. Ce chemin, les traducteurs de contrats l'ouvrent tout grand lorsqu'ils en font la publicité dans leurs textes. En revanche, ils ont également le pouvoir de l'obstruer, en mettant en valeur les équivalents propres au droit civil (traduction cibliste). Cette mission peut paraître délicate pour certains, mais elle n'a rien d'impossible, ni même d'excessivement difficile. 


\section{La mission possible du traducteur de contrats}

Le traducteur de contrats qui opte pour une méthode cibliste devra forcément déployer plus d'efforts que celui qui se complaît dans une approche sourcière. Comme les sourciers procèdent par équivalence formelle (Ladmiral, 2014 [1986], p.9), il leur suffit de consulter TERMIUM Plus pour obtenir la version française officielle des termes de common law à traduire. L'équivalence sera rigoureusement exacte sur le plan formel puisque ces termes ont été normalisés par le PAJLO, en anglais comme en français. Les ciblistes, quant à eux, ne peuvent prendre ce raccourci, car ils doivent «comparer le droit exprimé dans la langue source au droit cible, avant de restituer le texte juridique dans la langue du droit cible» (Monjean-Decodin, 2010, p. 702).

Cet exercice de droit comparé est de nature à semer le découragement chez le traducteur. En effet, la traduction transsystémique d'un contrat est souvent présentée comme une épreuve périlleuse, voire insurmontable, au motif que «les institutions de chaque système ne peuvent être comparées à celles de l'autre» (Gémar, 1980, p. 148). On a souligné notamment la grande complexité inhérente à la traduction d'un contrat de l'anglais au chinois (Cao, 1997). Même la traduction des concepts du droit des contrats de l'allemand au français a posé des difficultés notables du fait des différences existant entre les cultures juridiques en présence, qui sont pourtant toutes deux de tradition civiliste (Lardeux, 2012). En ce qui concerne le passage de la common law au droit civil, les écueils peuvent paraître infranchissables (Gémar, 2015, p. 482).

Dès lors, les traducteurs peuvent être tentés de déserter le terrain miné que représente la traduction de contrats de l'anglais au français. Aussi est-il important de les rassurer en relativisant la situation. Tout d'abord, il faut savoir que les termes proprement juridiques ne forment habituellement qu'une infime fraction du texte d'un contrat. Les compétences linguistiques générales du traducteur se révèlent donc, pour la plus grande part de son travail, bien plus importantes que ses connaissances juridiques. Ensuite, les attentes envers les traducteurs de contrats sont censées être raisonnables et tenir compte du fait que ceux-ci «se situent, le plus souvent, à l'extérieur des groupes socioprofessionnels dont émanent [1]es textes» (Collet, 2014, p. 123). Il serait déraisonnable de demander aux langagiers d'être des experts du domaine de spécialité dans lequel ils traduisent, en l'occurrence le droit des contrats. On ne pourra jamais reprocher au traducteur, 
par exemple, de ne pas avoir remarqué qu'une clause était invalide en droit québécois et aurait par conséquent dû être rayée du contrat.

Par-dessus tout, il importe d'éviter le piège que constitue la soidisant intraduisibilité du droit. Le mythe est coriace. Gémar a récemment souligné qu'encore aujourd'hui, «[d]e nombreux traductologues et juristes croient que la traduction juridique est, sinon impossible, du moins problématique. Ils pensent qu'une traduction ne reproduit pas à l'identique les effets juridiques du texte de départ» (2016, p. 533). Leur conclusion, soit l'infaisabilité de la traduction juridique, est erronée, mais leur prémisse n'en est pas moins incontestable. À n'en point douter, un contrat issu d'un territoire de common law qui est traduit conformément à la terminologie civiliste ne produira pas les mêmes effets juridiques dans la langue d'arrivée que dans la langue de départ. Ils font toutefois fausse route s'ils attribuent cette situation au processus de traduction. Si les effets juridiques diffèrent d'une version du contrat à l'autre, ce n'est pas parce que les termes du sacro-saint texte source ont été déformés par le traducteur, mais tout simplement parce que la législation applicable n'est pas la même. Cet état de fait n'a rien de problématique. C'est plutôt la situation inverse qui le serait. Si un contrat produisait exactement le même effet des deux côtés d'une frontière, ce serait le signe que l'un des deux États a abandonné sa souveraineté, bref la fin de la démocratie.

En clair, la traduction transsystémique d'un contrat ne consiste nullement à produire une version française conforme en tous points à la version anglaise. À l'impossible nul n'est tenu, pas même le traducteur d'un contrat de plusieurs millions de dollars... Les équivalents civilistes utilisés dans le texte d'arrivée ne seront sans doute pas des équivalents parfaits des termes de common law de départ, mais ils conviendront néanmoins parfaitement dans le nouveau contexte d'énonciation! Gémar fournit un exemple incontournable, résidant dans la notion de contrat elle-même : "On sait que le contract de la common law n'est pas le contrat du droit français» (2015, p. 482). On ne s'arrêtera toutefois pas à cette absence de correspondance parfaite, sans quoi même le titre du document se révélerait intraduisible, ce qui serait une grossière absurdité. En pratique, les nuances distinguant les deux concepts ne sauraient empêcher le traducteur de rendre contract par «contrat»... C'est pourquoi Gémar ajoute ce qui suit :

En outre, quand il s'agit de traduire le droit, il est vain de chercher une équivalence parfaite. Lenveloppe linguistique du concept est somme 
toute secondaire; ce qui compte, c'est non pas l'équivalence des concepts, mais celle des textes. (ibid.)

C'est dans cette perspective pragmatique que le juge LouisPhilippe Pigeon, pionnier de la jurilinguistique, a recommandé aux traducteurs juridiques d'adopter la méthode dite de l'équivalence fonctionnelle, dans un texte publié en 1982 et cité de nombreuses fois depuis. L'équivalence fonctionnelle, également connue sous le nom d'équivalence dynamique, peut se définir par opposition à l'équivalence formelle (Nida, 1964). La méthode de l'équivalence formelle, qui est encore fréquemment imposée aux traducteurs juridiques, exige la fidélité linguistique, c'est-à-dire que le texte cible doit être une reproduction rigoureusement exacte du texte source (Harvey, 2002, p. 181). Comme son nom l'indique, la méthode de l'équivalence fonctionnelle met plutôt l'accent sur la fonction du texte ou du terme à traduire. Dans un contexte juridique, l'équivalent fonctionnel d'un terme du système juridique source est le terme qui remplit la même fonction dans le système juridique cible (Sarcevic, 2000, p. 236).

En définitive, le traducteur qui, dans un contrat, est confronté à une notion de common law doit «rechercher son but et l'équivalence fonctionnelle, en droit civil, de l'institution de common law visée» (Groffier, 1990, p. 318). Les écrits de Gémar permettent encore une fois d'illustrer le principe. Parmi les cas d'équivalence fonctionnelle qu'il énumère, on peut citer l'exemple caractéristique du couple «mortgage/ hypothèque»(Gémar, 2002, p.170). D’un point de vue notionnel, la différence entre les deux termes est de taille : le «mortgage» de common law entraîne un transfert du titre de propriété en faveur du créancier, tandis que "l'hypothèque» de droit civil n'a pas cet effet (Emerich, 2010, p. 133). Malgré cette distinction fondamentale en ce qui concerne le régime juridique applicable, les deux termes décrivent la même réalité pratique : un bien tient lieu de garantie contre le risque de non-paiement d'une dette. L'un est donc l'équivalent fonctionnel de l'autre.

Un autre exemple fourni par le même auteur permet d'approfondir la réflexion. Gémar laisse entendre qu'il n'y a pas d'équivalence fonctionnelle entre les termes "act of God» et «force majeure» "parce que les notions ne se recoupent pas» (2002, p.171). Cette opinion est contestable malgré l'importante différence sémantique existant effectivement entre les termes. Il est vrai qu'en common law, la notion d'«acte de Dieu», ou d'«acte de la nature» dans sa version 
moderne normalisée par le PAJLO, ne comprend pas le fait d'un tiers, contrairement à la «force majeure» du droit civil (Groffier, 1990, p. 317). Il n'y a donc manifestement pas équivalence conceptuelle, de sorte qu'il est trompeur d'établir une relation de synonymie entre les termes «acte de Dieu» et «force majeure » comme le fait Hubert Reid dans son dictionnaire (2016). Il est toutefois logique de conclure à l'équivalence fonctionnelle, car les termes visent tous deux à prévoir quelle sera la responsabilité de l'une et l'autre des parties advenant un problème indépendant de leur volonté. Les termes "act of God» et «force majeure» remplissent la même fonction même si dans certaines circonstances - les cas où un tiers est impliqué -, la clause n'aura pas le même effet dans la version traduite que dans la version originale. Il est toutefois préférable qu'il en soit ainsi... Si le concept anglo-saxon était rendu par le terme "acte de la nature», étranger au droit civil, la portée de la clause au Québec serait fort douteuse. Bien sûr, pour des raisons commerciales, d'aucuns pourraient préférer la conception anglaise. Dans un tel cas, la solution ne sera toujours pas d'utiliser le terme du PAJLO, mais bien de restreindre expressément dans le contrat la portée de la notion de force majeure en précisant qu'elle exclut l'intervention d'un tiers. Il n'appartient toutefois pas au traducteur d'apporter ce type de modification au contrat. S'il a utilisé l'équivalent fonctionnel "force majeure», le traducteur pourra dire mission accomplie.

Voilà la recette pour traduire un contrat conformément à l'approche cibliste. En résumé, chaque notion de common law rencontrée dans le texte doit déclencher une quête visant à trouver un équivalent fonctionnel dans le lexique du droit civil. Telle est la mission - fort stimulante! - confiée au traducteur de contrats.

\section{Conclusion}

En matière de traduction de contrats, le débat traductologique classique opposant les sourciers et les ciblistes a bien plus qu'une valeur théorique. Il touche en réalité l'ensemble de la société. Concrètement, l'approche sourcière met en péril le système juridico-politique propre au Québec, une richesse inouïe du point de vue de la diversité culturelle. À l'échelle individuelle, ce procédé de traduction ne représente certes qu'une pratique machinale, dénuée de toute intention politique malveillante, mais dans une perspective globale, il participe d'un anéantissement politique du système juridique du Québec. 
Pour conclure sur une note optimiste, l'écroulement définitif du vocabulaire du droit civil sous le poids de la common law peut encore être évité. La vitalité de ce vocabulaire repose bien sûr en partie sur les titulaires de pouvoirs publics, mais il faut se garder de minimiser la contribution que les traducteurs de contrats peuvent apporter. Il leur suffit de privilégier la terminologie civiliste locale lorsque vient le temps de rendre un concept de common law dans un contrat traduit pour des Québécois. De cette façon, ils ont le pouvoir de promouvoir la culture juridique unique du Québec.

Ce pouvoir est avant tout politique, car il s'agit de la préservation des traits distinctifs d'un peuple minoritaire dans un contexte politique défavorable. Malgré l'hégémonie que le Canada anglais exerce depuis la conquête de la Nouvelle-France sur la seule nation francophone d'Amérique du Nord, son régime civiliste de tradition française est toujours vivant, et il le demeurera si les différents acteurs de la société unissent leurs efforts. Chaque geste, aussi minime soit-il, allant dans le sens du maintien de ce riche héritage mérite d'être souligné. Le travail des traducteurs de contrats ne fait pas exception. Levons notre chapeau à tous ceux qui prennent soin de respecter la spécificité juridique du Québec dans leurs traductions.

\section{Références}

Arbour, Marie-Ève (2012). Fragments de droit québécois et canadien - Histoire, mixité, mutations. Cowansville, Éditions Yvon Blais.

Baudouin, Jean-Louis (1996). "Quelques réflexions d'un civiliste sur la common law plurielle ». Revue de la common law en français, 1, 2, p. 283-290.

Berman, Antoine (1984). L'épreuve de l'étranger. Paris, Gallimard.

Brun, Pierre (1993). «La pollution du partage des compétences par le droit de l'environnement». Revue générale de droit, 24, 2, p. 191-225.

Cachecho, Maya (2015). Les droits réels et personnels sur les titres détenus auprès d'un intermédiaire: analyse comparative en droit québécois interne et international privé. Thèse de doctorat, Département de droit, Université de Montréal.

Cao, Deborah (1997). «Consideration in Translating English/Chinese Contracts». Meta, 42, 4, p. 661-669.

Collet, Tanja (2014). «Obstacles lexico-sémantiques à la lecture réussie d'un texte de spécialité». TTR, 27, 1, p. 123-148.

Cordonnier, Jean-Louis (2002). «Aspects culturels de la traduction : quelques notions clés». Meta, 47, 1, p. 38-50.

Cornu, Gérard (1995). «Français juridique et science du droit: synthèse». In G. Snow et J. Vanderlinden, dir. Français juridique et science du droit. Bruxelles, Bruylant, p. 11-19. 
Cornu, Gérard (2005). Linguistique juridique, $3^{\mathrm{e}}$ éd. Paris, Montchrestien.

Dalphond, Pierre J. (2003). «Le style civiliste et le juge : le juge québécois ne serait-il pas le prototype du juge civiliste de l'avenir?». In N. Kasirer, dir. Le droit civil, avant tout un style? Montréal, Thémis, p. 81-100.

Debruche, Anne-Françoise (2015). «La tradition romaniste, une espèce menacée? Libre propos sur le mythe du droit civil inutile et abstrait». Les Cabiers de droit, 56, 1, p. 3-33.

Delisle, Jean (2001). «L'évaluation des traductions par l'historien ». Meta, 46, 2, p. 209-226.

Delisle, Jean et Marco A. Fiola (2013). La traduction raisonnée. Manuel d'initiation à la traduction professionnelle de l'anglais vers le français, $3^{\mathrm{e}}$ éd. Ottawa, Presses de l'Université d'Ottawa.

Després, Christian C. (2007). «L'enseignement de la traduction juridique au deuxième cycle». Circuit, 95, p. 6-7.

Dullion, Valérie (2014). «Traduire les textes juridiques dans un contexte de plurilinguisme officiel: quelle formation pour quelles compétences spécifiques?». Meta, 59, 3, p. 636-653.

Emerich, Yaell (2010). «La nature juridique des sûretés réelles en droit civil et en common law: une question de tradition juridique?». Revue juridique Thémis, 44, 1, p. 95-140.

Ferreira Duarte, João (2000). «The Politics of Non-Translation: A Case Study in Anglo-Portuguese Relations». TTR, 13, 1, p. 95-112.

Garner, Bryan A. et Henry Campbell Black (2014). Black's Law Dictionary, 10 éd. St. Paul, MN, Thomson Reuters.

Gémar, Jean-Claude (1980). «Le traducteur et la documentation juridique». Meta, 25, 1, p. 134-151.

Gémar, Jean-Claude (1982). «Fonctions de la traduction juridique en milieu bilingue et langage du droit au Canada.» In J.-C. Gémar, dir. Langage du droit et traduction. Essais de jurilinguistique. Montréal, Conseil de la langue française, n. p.

Gémar, Jean-Claude (1988). «La traduction juridique: art ou technique d'interprétation?». Meta, 33, 2, p. 304-318.

Gémar, Jean-Claude (2002). «Le plus et le moins-disant culturel du texte juridique. Langue, culture et équivalence». Meta, 47, 2, p. 163-176.

Gémar, Jean-Claude (2005). «De la traduction (juridique) à la jurilinguistique. Fonctions proactives du traductologue». Meta, 50, 4, n. p.

Gémar, Jean-Claude (2015). «De la traduction juridique à la jurilinguistique : la quête de l'équivalence». Meta, 60, 3, p. 476-493.

Gémar, Jean-Claude (2016). "Chronique bibliographique : Gérard Cornu, Dictionary of the Civil Code». Les Cabiers de droit, 57, 3, p. 532-539.

Gémar, Jean-Claude et Vo Ho-Thuy (2016). Nouvelles difficultés du langage du droit au Canada. Montréal, Thémis.

Gémar, Jean-Claude et Nicholas Kasirer, dir. (2005). Jurilinguistique : entre langues et droits, Montréal, Thémis. 
Gómez, Carmen et María Pinto (2001). «La normalisation au service du traducteur». Meta, 46, 3, p. 564-579.

Gouvernement du Canada (n. d.). TERMIUM Plus. [http://www.btb. termiumplus.gc.ca/tpv2alpha/alpha-fra.html?lang=fra].

Groffier, Ethel (1990). «La langue du droit». Meta, 35, 2, p. 314-331.

Harvey, Malcolm (2002). «What's so Special about Legal Translation?». Meta, 47,2 , p. $177-185$.

Jobin, Pierre-Gabriel (2000). «La Cour suprême et la réforme du Code Civil». Canadian Bar Review, 79, 2, p. 27-46.

Koutsivitis, Vassilis (1990). «La traduction juridique : standardisation versus créativité». Meta, 35, 1, p. 226-229.

Ladmiral, Jean-René (2014a). «Avant-propos». dans Sourcier ou cibliste. Paris, Les Belles Lettres, p. XI à XIX.

Ladmiral, Jean-René (2014 [1986]). «Sourciers et ciblistes». In Sourcier ou cibliste. Paris, Les Belles Lettres, p. 3-27.

Ladmiral, Jean-René (2014 [1990]). «Pour une théologie de la traduction ». In Sourcier ou cibliste. Paris, Les Belles Lettres, p. 257-280.

Ladmiral, Jean-René (2014 [1991]). «Vers une métaphysique de la traduction ». In Sourcier ou cibliste. Paris, Les Belles Lettres, p. 225-256.

Ladmiral, Jean-René (2014 [1998]). «Dialectique du littéralisme». In Sourcier ou cibliste. Paris, Les Belles Lettres, p. 175-223.

Lardeux, Gwendoline (2012). «Terminologie et traduction des législations relatives au droit des contrats. L'exemple du BGB». Revue internationale de droit comparé, 64, 3, p. 817-839.

Lavoie, Judith (2003). "Faut-il être juriste ou traducteur pour traduire le droit?". Meta, 483, p. 393-401.

LeBel, Louis et Pierre-Louis Le Saunier (2006). "L'interaction du droit civil et de la common law à la Cour suprême du Canada». Les Cabiers de droit, 47, 2, p. 179-238.

Lehman, Jeffrey et Shirelle Phelps (2005). West's Encyclopedia of American Law, $2^{\mathrm{e}}$ éd. Detroit, Gale.

Meschonnic, Henri (1973). Pour la poétique II. Paris, Gallimard, 1973.

Monjean-Decaudin, Sylvie (2010). «Territorialité et extraterritorialité de la traduction du droit». Meta, 55, 4, p. 693-711.

Moore, Benoît (2007). "L'acculturation en droit de la consommation québécois». In J.-L. Navarro et G. Lefebvre, dir. L'acculturation en droit des affaires, Montréal, Thémis, p. 295-326.

Nida, Eugene A. (1964). Toward a Science of Translating. Leyde, Brill.

Nord, Christiane (1997). Translating as a Purposeful Activity: Functionalist Approaches Explained. Manchester, St. Jerome.

Normand, Sylvio (2011). «La culture juridique et l'acculturation du droit : le Québec ", ISAIDAT Law Review, volume 1, numéro spécial 1, article 23.

Pigeon, Louis-Philippe (1982), «La traduction juridique - L'équivalence fonctionnelle». In J.-C. Gémar, dir. Langage du droit et traduction - Essais de 
jurilinguistique. Montréal, Conseil de la langue française, n. p.

Poirier, Éric (2014). La Charte de la langue française et l'abandon des moyens pour atteindre son objectif. Institut de recherche sur le Québec.

Popovici, Adrian (2000), «Le rôle de la Cour suprême en droit civil». Revue juridique Thémis, 34, p. 607-624.

Popovici, Adrian (2009). «Libres propos sur la culture juridique québécoise dans un monde qui rétrécit». Revue de droit de l'Université McGill, 54, 2, p. 223-236.

Raimondo, Riccardo (2016). "Orphée contre Hermès : traduction, herméneutique et imaginaire». Meta, 61, 3, p. 650-674.

Reed, David G. (1979). «Problèmes de la traduction juridique au Québec». Meta, 241, p. 95-102.

Reid, Hubert (2016). Dictionnaire de droit québécois et canadien. Montréal, Wilson \& Lafleur.

Sabourin, Frédérique (2013). «L'harmonisation des lois provinciales et territoriales canadiennes et le droit québécois». Revue de droit de l'Université de Sherbrooke, 43, p. 511-560.

Salinas, Bestué C. (2010). "Les clauses d'exonération de responsabilité : stratégies de traduction ». Meta, 55, 2, p. 287-308.

Sarcevic, Susan (2000). New Approach to Legal Translation. La Haya, Kluwer Law International.

Simon, Sherry (1989). «Compte rendu de «La Traduction», Jean-René Ladmiral, Revue d'esthétique, nouvelle série, 12, 1986 ». TTR, 2, 2, p. 157158.

Sparer, Michel (1979). «Pour une dimension culturelle de la traduction juridique». Meta, 24, 1, p. 68-94.

Sparer, Michel (2002). «Peut-on faire de la traduction juridique? Comment doit-on l'enseigner?». Meta, 47, 2, p. 266-278.

Tellier-Marcil, Arnaud (2016). La rédaction en droit des affaires : principes fondamentaux et recommandations pratiques. Montréal, Éditions Yvon Blais.

Touchette, Josée (2002). «Le bijuridisme canadien: coexistence de deux systèmes juridiques institutionnels». Revue générale de droit, 32, 1, p. 117130.

Tremblay, Guy (2000). «La version française des lois constitutionnelles du Canada». Les Cahiers de droit, 41, 1, p. 33-60.

Vanderlinden, Jacques, Gérard Snow et Donald Poirier (2017). La common law de $A$ à Z. $2^{\mathrm{e}}$ éd. Montréal, Éditions Yvon Blais.

Arnaud Tellier-Marcil

Faculté de droit

Université de Montréal

Montréal (Québec), Canada arnaud.marcil@gmail.com 